\title{
OPTIMIZED ROBUST CONTROL INVARIANT SETS FOR CONSTRAINED LINEAR DISCRETE-TIME SYSTEMS *
}

\author{
S. V. Raković ${ }^{* 1}$ D. Q. Mayne ${ }^{*}$ E. C. Kerrigan ${ }^{* *}$ \\ K. I. Kouramas * \\ * Imperial College London, London SW7 2BT, United \\ Kingdom. Tel: +44-(0)20-7594-6295/87/81. Fax: \\ +44-(0)20-7594-6282, E-mail: \\ sasa.rakovic@imperial.ac.uk,d.mayne@imperial.ac.uk and \\ k.kouramas@imperial.ac.uk \\ ** Royal Academy of Engineering Post-doctoral Research \\ Fellow; Department of Engineering, University of \\ Cambridge, Trumpington Street, Cambridge CB2 1PZ, \\ United Kingdom. Tel: +44-(0)1223-332600. Fax: \\ +44-(0)1223-332662. Email: erickerrigan@ieee.org
}

\begin{abstract}
In this paper we introduce the concept of optimized robust control invariance for a discrete-time, linear, time-invariant system subject to additive state disturbances. A novel characterization of a family of the robust control invariant sets is given. The existence of a constraint admissible member of this family can be checked by solving a single linear programming problem. The solution of the same linear programming problem yields the corresponding feedback controller. Copyright ${ }^{\circledR} 2005$ IFAC.
\end{abstract}

Keywords: Set invariance, Constrained control, Linear systems.

\section{INTRODUCTION}

The theory of set invariance plays a fundamental role in the control of constrained systems. The interested reader is referred to the important and comprehensive survey paper (Blanchini, 1999) for an introduction to set invariance and a number of relevant references. Two important issues, the computation of the minimal robust positively invariant (mRPI) set and the maximal robust positively invariant (MRPI), set are studied in detail in (Kolmanovsky and Gilbert, 1998).

\footnotetext{
* Research supported by the Engineering and Physical Sciences Research Council, UK and the Royal Academy of Engineering, UK.

1 Corresponding Author.
}

From the control theory point of view, set invariance provides useful tools for the synthesis of reference governors (Gilbert and Kolmanovsky, 1999) and predictive controllers (Bemporad and Morari, 1999; Findeisen et al., 2003; Mayne, 2001) with guaranteed invariance, stability and convergence properties. Since the mRPI set is the smallest invariant set for a system, it is also a suitable target set in robust time-optimal control (Bertsekas and Rhodes, 1971; Blanchini, 1992; Mayne and Schroeder, 1997) and plays an integral part in a novel robust predictive control method recently proposed in (Langson et al., 2004).

It is the main purpose of this paper to provide a novel characterization of a family of the polytopic robust control invariant sets. Verifying existence 
of a constraint admissible member of this family as well as the computation of the corresponding feedback controller can be efficiently realized by solving a single linear programming problem (LP). This paper is organized as follows. Section 2 is concerned with the preliminaries. Section 3 addresses the robust control invariance issue. Section 4 provides an interesting comparison to existing methods. Finally, Section 5 indicates possible applications of the results and presents conclusions.

Notation: Let $\mathbb{N} \triangleq\{0,1,2, \ldots\}, \mathbb{N}_{+} \triangleq\{1,2, \ldots\}$ and $\mathbb{N}_{q} \triangleq\{0,1, \ldots, q\}$. Let $\mathbf{1}_{t}$ denote the vector $(1,1, \ldots, 1)^{\prime} \in \mathbb{R}^{t}$. Let $\operatorname{abs}(A)$ denote the matrix whose elements are the absolute values of the corresponding components of the matrix $A$. Given two matrices $A$ and $B, \operatorname{vec}(A)$ denotes standard stack operator and $A \otimes B$ is the Kronecker product of matrices $A$ and $B$. A polyhedron is the (convex) intersection of a finite number of open and/or closed half-spaces and a polytope is the closed and bounded polyhedron. Let $\mathbb{B}_{p}^{n}(r) \triangleq\left\{x \in \mathbb{R}^{n} \mid\right.$ $\left.|x|_{p} \leq r\right\}$ be a $p$-norm ball in $\mathbb{R}^{n}$, where $r \geq 0$ and $|\cdot|_{p}$ denotes the vector $p$-norm. Given two sets $\mathcal{U}$ and $\mathcal{V}$, such that $\mathcal{U} \subset \mathbb{R}^{n}$ and $\mathcal{V} \subset \mathbb{R}^{n}$, the Minkowski (vector) sum is defined by $\mathcal{U} \oplus \mathcal{V} \triangleq\{u+$ $v \mid u \in \mathcal{U}, v \in \mathcal{V}\}$. Given the sequence of sets $\left\{\mathcal{U}_{i} \subset \mathbb{R}^{n}\right\}_{i=a}^{b}$, we define $\bigoplus_{i=a}^{b} \mathcal{U}_{i} \triangleq \mathcal{U}_{a} \oplus \cdots \oplus \mathcal{U}_{b}$. The support function of a set $\Pi \subset \mathbb{R}^{n}$, evaluated at $z \in \mathbb{R}^{n}$, is defined as $h(\Pi, z) \triangleq \sup _{\pi \in \Pi} z^{T} \pi$.

\section{PRELIMINARY DEFINITIONS AND EXISTING RESULTS}

We consider the following discrete-time linear time-invariant (DLTI) system:

$$
x^{+}=A x+B u+w,
$$

where $x \in \mathbb{R}^{n}$ is the current state, $u \in \mathbb{R}^{m}$ is the current control action $x^{+}$is the successor state, $w \in \mathbb{R}^{n}$ is an unknown disturbance and $(A, B) \in \mathbb{R}^{n \times n} \times \mathbb{R}^{n \times m}$. The disturbance $w$ is persistent, but contained in a convex and compact set $W \subset \mathbb{R}^{n}$ that contains the origin. We make the standing assumption that the couple $(A, B)$ is controllable.

The system (2.1) is subject to the following set of hard state and control constraints:

$$
(x, u) \in X \times U
$$

where $X \subseteq \mathbb{R}^{n}$ and $U \subseteq \mathbb{R}^{m}$ are polyhedral and polytopic sets respectively and both contain the origin as an interior point.

Most of the previous research considered the case $u=\mu(x)=K x$ and the corresponding autonomous DLTI system:

$$
x^{+}=A_{K} x+w, A_{K} \triangleq(A+B K),
$$

where $A_{K} \in \mathbb{R}^{n \times n}$ and all the eigenvalues of $A_{K}$ are strictly inside the unit disk. Given any $K \in \mathbb{R}^{m \times n}$ let $X_{K} \triangleq\{x \mid x \in X, K x \in U\} \subset \mathbb{R}^{n}$.

Definition 1. The set $\Omega \subset \mathbb{R}^{n}$ is a robust positively invariant (RPI) set for the system (2.3) and constraint set $\left(X_{K}, W\right)$ if $\Omega \subseteq X_{K}$ and $A_{K} x+$ $w \in \Omega$ for all $x \in \Omega$ and all $w \in W$.

Definition 2. The minimal robust positively invariant (mRPI) set $F_{\infty}$ for the system (2.3) and constraint set $\left(\mathbb{R}^{n}, W\right)$ is the RPI set for the system $(2.3)$ and constraint set $\left(\mathbb{R}^{n}, W\right)$ that is contained in every closed, RPI set for the system (2.3) and constraint set $\left(\mathbb{R}^{n}, W\right)$.

The mRPI set $F_{\infty}$ exists, is unique, compact and contains the origin (Kolmanovsky and Gilbert, 1998, Sect. IV). The mRPI set $F_{\infty}$ is the limit of the set sequence $\left\{F_{i}\right\}$ defined by:

$$
F_{i} \triangleq \bigoplus_{j=0}^{i-1} A_{K}^{j} W, i \in \mathbb{N}_{+} \text {and } F_{0} \triangleq\{0\}
$$

The mRPI set is then given by:

$$
F_{\infty}=\operatorname{closure}\left(\bigoplus_{i=0}^{\infty} A_{K}^{i} W\right)
$$

It is impossible in general to obtain an explicit characterization of the mRPI set $F_{\infty}$. In (Raković et al., 2005) a method for computation of an $\varepsilon$ $(\varepsilon>0)$ outer RPI approximation of the mRPI $F_{\infty}$ is given:

Theorem 1. If $0 \in \operatorname{interior}(W)$, then for all $\varepsilon>0$, there exists $\zeta \in[0,1)$ and a corresponding integer $s$ such that the following set inclusions

$$
A_{K}^{s} W \subseteq \zeta W \text { and } \zeta(1-\zeta)^{-1} F_{s} \subseteq \mathbb{B}_{p}^{n}(\varepsilon)
$$

are true. Furthermore, if (2.6) is satisfied, then the set $F_{(\zeta, s)}$ defined by:

$$
F_{(\zeta, s)} \triangleq(1-\zeta)^{-1} F_{s}
$$

where $F_{i}$ is defined by (2.4), is an RPI set for the system $(2.3)$ and constraint set $\left(\mathbb{R}^{n}, W\right)$ such that $F_{\infty} \subseteq F_{(\zeta, s)} \subseteq F_{\infty} \oplus \mathbb{B}_{p}^{n}(\varepsilon)$.

This result can be extended to case when the origin is in the relative interior of $W$ (Rakovic, 2005).

Definition 3. The set $\Omega \subset \mathbb{R}^{n}$ is a robust control invariant (RCI) set for the system (2.1) and constraint set $(X, U, W)$ if $\Omega \subseteq X$ and for all $x \in \Omega$ there exists a $u \in U$ such that $A x+B u+$ $w \in \Omega$ for all $w \in W$.

An RPI set for the system (2.3) and constraint set $\left(X_{K}, W\right)$ exists if and only if $F_{\infty} \subseteq X_{K}$; this con- 
dition is not necessarily satisfied for an arbitrary selected stabilizing feedback controller $K$. In this note we provide a method for checking existence of a RCI set for the system (2.1) and constraint set $(X, U, W)$ as well as the computation of the corresponding control policy via an optimization procedure.

\section{ROBUST CONTROL INVARIANCE ISSUE}

Let $M_{i} \in \mathbb{R}^{m \times n}, i \in \mathbb{N}$ and for each $k \in \mathbb{N}$ let $\mathbf{M}_{k} \triangleq\left(M_{0}, M_{1}, \ldots, M_{k-2}, M_{k-1}\right)$. An appropriate characterization of a family of RCI sets for the system (2.1) and constraint set $\left(\mathbb{R}^{n}, \mathbb{R}^{m}, W\right)$ is given by the following sets for $k \geq n$ :

$$
R_{k}\left(\mathbf{M}_{k}\right) \triangleq \bigoplus_{i=0}^{k-1} D_{i}\left(\mathbf{M}_{k}\right) W
$$

where the matrices $D_{i}\left(\mathbf{M}_{k}\right), i \in \mathbb{N}_{k}, k \geq n$ are defined by:

$D_{0}\left(\mathbf{M}_{k}\right)=I, D_{i}\left(\mathbf{M}_{k}\right) \triangleq A^{i}+\sum_{j=0}^{i-1} A^{i-1-j} B M_{j}, i \geq 1$

providing that $\mathbf{M}_{k}$ satisfies:

$$
D_{k}\left(\mathbf{M}_{k}\right)=\mathbf{0}
$$

Since the couple $(A, B)$ is assumed to be controllable, such a choice exists for all $k \geq n$. Let $\mathbb{M}_{k}$ denote the set of all matrices $\mathbf{M}_{k}$ satisfying condition (3.3):

$$
\mathbb{M}_{k} \triangleq\left\{\mathbf{M}_{k} \mid D_{k}\left(\mathbf{M}_{k}\right)=\mathbf{0}\right\}
$$

Theorem 2. (Raković, 2005) Given any $\mathbf{M}_{k} \in$ $\mathbb{M}_{k}, \quad k \geq n$ and the corresponding set $R_{k}\left(\mathbf{M}_{k}\right)$ there exists a control law $\mu: R_{k}\left(\mathbf{M}_{k}\right) \rightarrow \mathbb{R}^{m}$ such that $A x+B \mu(x) \oplus W \subseteq R_{k}\left(\mathbf{M}_{k}\right), \forall x \in R_{k}\left(\mathbf{M}_{k}\right)$, i.e. the set $R_{k}\left(\mathbf{M}_{k}\right)$ is RCI for the system (2.1) and constraint set $\left(\mathbb{R}^{n}, \mathbb{R}^{m}, W\right)$.

The feedback control law $\mu: R_{k}\left(\mathbf{M}_{k}\right) \rightarrow \mathbb{R}^{m}$ in Theorem 2 is a selection from the set valued map:

$$
\mathcal{U}(x) \triangleq \mathbf{M}_{k} \mathbf{W}(x)
$$

where $\mathbf{M}_{k} \in \mathbb{M}_{k}$ and the set of disturbance sequences $\mathbf{W}(x)$ is defined for each $x \in R_{k}\left(\mathbf{M}_{k}\right)$ by:

$$
\mathbf{W}(x) \triangleq\left\{\mathbf{w} \mid \mathbf{w} \in \mathbf{W}^{k}, D \mathbf{w}=x\right\},
$$

where $\mathbf{W}^{k} \triangleq W \times W \times \ldots \times W$ and $D=$ $\left[D_{k-1}\left(\mathbf{M}_{k}\right) \ldots D_{0}\left(\mathbf{M}_{k}\right)\right]$. A $\mu(\cdot)$ satisfying Theorem 2 can be defined, for instance, as follows:

$$
\begin{aligned}
\mu(x) & \triangleq \mathbf{M}_{k} \mathbf{w}^{0}(x) \\
\mathbf{w}^{0}(x) & \triangleq \arg \min _{\mathbf{w}}\left\{|\mathbf{w}|_{2}^{2} \mid \mathbf{w} \in \mathbf{W}(x)\right\}
\end{aligned}
$$

The function $\mathbf{w}^{0}(\cdot)$ is piecewise affine, being the solution of a parametric quadratic programme; since the feedback control law $\mu: R_{k}\left(\mathbf{M}_{k}\right) \rightarrow \mathbb{R}^{m}$ is a linear map of a piecewise affine function it is piecewise affine.

Theorem 2 states that for any $k \geq n$ the RCI set $R_{k}\left(\mathbf{M}_{k}\right)$, finitely determined by $k$, is easily computed if $W$ is a polytope. The set $R_{k}\left(\mathbf{M}_{k}\right)$ is parametrized by the matrix $\mathbf{M}_{k}$; this allows us to formulate an LP that yields the set $R_{k}\left(\mathbf{M}_{k}\right)$ while minimizing an appropriate norm of the set $R_{k}\left(\mathbf{M}_{k}\right)$.

\subsection{Optimized Robust Control Invariance}

We provide a full exposition for the case when:

$$
W \triangleq\left\{E d+\left.f|| d\right|_{\infty} \leq \eta\right\}
$$

where $d \in \mathbb{R}^{t}, E \in \mathbb{R}^{n \times t}$ and $f \in \mathbb{R}^{n}$. We are interested in the computation of a RCI set $R_{k}\left(\mathbf{M}_{k}\right)$ for the system (2.1) and constraint set $\left(\mathbb{R}^{n}, \mathbb{R}^{m}, W\right)$ contained in a 'minimal' $p$-norm ball, i.e. we wish to find $R_{k}^{0}=R_{k}\left(\mathbf{M}_{k}^{0}\right)$ where:

$\left(\mathbf{M}_{k}^{0}, \alpha^{0}\right)=\arg \min _{\mathbf{M}_{k}, \alpha}\left\{\alpha \mid R_{k}\left(\mathbf{M}_{k}\right) \subseteq \mathbb{B}_{p}(\alpha), \alpha>0\right\}$

We show that our problem can be posed as an LP if $p=1, \infty$ by considering a more general problem:

$$
\mathbb{P}_{k}:\left(\mathbf{M}_{k}^{0}, \alpha^{0}\right)=\arg \min _{\mathbf{M}_{k}, \alpha}\left\{\alpha \mid\left(\mathbf{M}_{k}, \alpha\right) \in \Omega\right\}
$$

where

$$
\begin{aligned}
& \Omega \triangleq\left\{\left(\mathbf{M}_{k}, \alpha\right) \mid \mathbf{M}_{k} \in \mathbb{M}_{k}, R_{k}\left(\mathbf{M}_{k}\right) \subseteq P(\alpha),\right. \\
& \alpha>0\} \text {, }
\end{aligned}
$$

and $P(1)$ is a polytope that contains the origin in its interior so that $P(\alpha) \triangleq\left\{x \mid C_{p} x \leq\right.$ $\left.\alpha c_{p}\right\}, \alpha>0$ with $C_{p} \in \mathbb{R}^{q \times n}$ and $c_{p} \in \mathbb{R}^{q}$. Before proceeding we recall few preliminary and elementary results (Raković, 2005):

Proposition 1. Let $\Pi$ be a non-empty set in $\mathbb{R}^{n}$ and $\Psi=\left\{\psi \in \mathbb{R}^{n} \mid f_{i}^{T} \psi \leq g_{i}, i \in \mathbb{N}_{l}\right\}$, where $f_{i} \in \mathbb{R}^{n}, g_{i} \in \mathbb{R}$. Then, $\Pi \subseteq \Psi$ if and only if $h\left(\Pi, f_{i}\right) \leq g_{i}$ for all $i \in \mathbb{N}_{l}$.

Proposition 2. Let each matrix $L_{k} \in \mathbb{R}^{n \times m}$ and each $\Phi_{k}$ be a non-empty, compact set in $\mathbb{R}^{m}$ for all $k \in \mathbb{N}_{K}$. If $\Pi=\bigoplus_{k=0}^{K} L_{k} \Phi_{k}$, then $h(\Pi, z)=$ $\sum_{k=0}^{K} \max _{\phi \in \Phi_{k}}\left(z^{T} L_{k}\right) \phi$.

The fact that $\max _{d}\left\{\left.a^{\prime} d|| d\right|_{\infty} \leq \eta\right\}=\eta|a|_{1}$ (Horn and Johnson, 1985) allows one to establish the following result:

Proposition 3. Let matrices $A \in \mathbb{R}^{n \times n}, C \in$ $\mathbb{R}^{q \times n}, D \in \mathbb{R}^{n \times p}$ and $M \in \mathbb{R}^{p \times n}$ and let $w \in W$ 
where $W=\left\{E d+\left.f|| d\right|_{\infty} \leq \eta\right\}$ and $E \in \mathbb{R}^{n \times t}$ and $f \in \mathbb{R}^{n}$. Then

$$
\begin{aligned}
& \max _{w \in W} C(A+D M) w= \\
& \quad \eta \operatorname{abs}(C(A+D M) E) \mathbf{1}_{t}+C(A+D M) f
\end{aligned}
$$

where the maximization is taken row-wise. Moreover, there exists a matrix $L \in \mathbb{R}^{q \times t}$ such that

$$
-L \leq C(A+D M) E \leq L,
$$

where the inequality is element-wise, and the solution to (3.12) satisfies

$$
\max _{w \in W} C(A+D M) w=\eta L \mathbf{1}_{q}+C(A+D M) f
$$

Proposition 1 implies that the set inclusion $R_{k}\left(\mathbf{M}_{k}\right) \subseteq P(\alpha)$ is true if and only if:

$$
\max _{x \in R_{k}\left(\mathbf{M}_{k}\right)} C_{p} x \leq \alpha c_{p},
$$

where the maximization is taken row-wise. It follows from Propositions 2 and 3 that there exist a set of matrices $L_{i} \in \mathbb{R}^{q \times t}, i \in \mathbb{N}_{k-1}$ such that:

$$
\max _{x \in R_{k}\left(\mathbf{M}_{k}\right)} C_{p} x=\sum_{i=0}^{k-1}\left(\eta L_{i} \mathbf{1}_{t}+C_{p} D_{i}\left(\mathbf{M}_{k}\right) f\right)
$$

where $\Lambda_{k} \triangleq\left\{L_{0}, L_{1}, \ldots, L_{k-1}\right\}$ and each $L_{i}$ satisfies:

$$
-L_{i} \leq C_{p} D_{i}\left(\mathbf{M}_{k}\right) E \leq L_{i}, i \in \mathbb{N}_{k-1}
$$

Since each $D_{i}\left(\mathbf{M}_{k}\right)$ is affine in $\mathbf{M}_{k}$ it follows by the basic properties of the Kronecker product (in particular $\left.\operatorname{vec}(A B C)=\left(C^{\prime} \otimes A\right) \operatorname{vec}(B)\right)$ that the set inclusion $R_{k}\left(\mathbf{M}_{k}\right) \subseteq P(\alpha)$ can be expressed as a set of linear inequalities in $\left(\operatorname{vec}\left(\mathbf{M}_{k}\right), \operatorname{vec}\left(\Lambda_{k}\right), \alpha\right)$. The condition $\mathbf{M}_{k} \in \mathbb{M}_{k}$ is a set of linear equalities in $\left(\operatorname{vec}\left(\mathbf{M}_{k}\right), \operatorname{vec}\left(\Lambda_{k}\right), \alpha\right)$. Since the cost (of $\left.\mathbb{P}_{k}\right)$ is a linear function of $\left(\operatorname{vec}\left(\mathbf{M}_{k}\right), \operatorname{vec}\left(\Lambda_{k}\right), \alpha\right)$ we can state the following:

Proposition 4. The minimization problem $\mathbb{P}_{k}$ defined in (3.10) is a linear programming problem.

An LP formulation of the problem $\mathbb{P}_{k}$ is:

$$
\mathbb{P}_{k}: \quad \min _{\gamma}\{\alpha \mid \gamma \in \Gamma\}
$$

where $\gamma \triangleq\left(\operatorname{vec}\left(\mathbf{M}_{k}\right), \operatorname{vec}\left(\Lambda_{k}\right), \alpha\right)$ and:

$$
\begin{gathered}
\Gamma \triangleq\left\{\gamma \mid \sum_{i=0}^{k-1}\left(\eta L_{i} \mathbf{1}_{t}+C_{p} D_{i}\left(\mathbf{M}_{k}\right) f\right) \leq \alpha c\right. \\
-L_{i} \leq C_{p} D_{i}\left(\mathbf{M}_{k}\right) E \leq L_{i}, \quad i \in \mathbb{N}_{k-1} \\
\left.\mathbf{M}_{k} \in \mathbb{M}_{k}, \alpha>0\right\}
\end{gathered}
$$

\subsection{Optimized Robust Control Invariance Under Constraints}

In this case it is possible to formulate an LP, whose feasibility establishes existence of a RCI set
$R_{k}\left(\mathbf{M}_{k}\right)$ for the system (2.1) and constraint set $(X, U, W)$. The control law $\mu(x)$ satisfies $\mu(x) \in$ $U\left(\mathbf{M}_{k}\right)$ for all $x \in R_{k}\left(\mathbf{M}_{k}\right)$ where:

$$
U\left(\mathbf{M}_{k}\right) \triangleq \bigoplus_{i=0}^{k-1} M_{i} W
$$

The state and control constraints (2.2) are satisfied if:

$$
R_{k}\left(\mathbf{M}_{k}\right) \subseteq \alpha X, U\left(\mathbf{M}_{k}\right) \subseteq \beta U
$$

where $\alpha X \triangleq\left\{x \mid C_{x} x \leq \alpha c_{x}\right\}, \beta U \triangleq\left\{u \mid C_{u} u \leq\right.$ $\left.\beta c_{u}\right\}$, (with $C_{x} \in \mathbb{R}^{q_{x} \times \bar{n}}, c_{x} \in \mathbb{R}^{q_{x}}, C_{u} \in \mathbb{R}^{q_{u} \times n}$, $\left.c_{u} \in \mathbb{R}^{q_{u}}\right)$ and $(\alpha, \beta) \in[0,1] \times[0,1]$.

Let now:

$$
\begin{aligned}
\bar{\Omega} \triangleq\left\{\left(\mathbf{M}_{k}, \alpha, \beta, \delta\right)\right. & \mid \mathbf{M}_{k} \in \mathbb{M}_{k}, R_{k}\left(\mathbf{M}_{k}\right) \subseteq \alpha X, \\
& U\left(\mathbf{M}_{k}\right) \subseteq \beta U, \\
& (\alpha, \beta) \in[0,1] \times[0,1], \\
& \left.q_{\alpha} \alpha+q_{\beta} \beta \leq \delta\right\}
\end{aligned}
$$

where $R_{k}\left(\mathbf{M}_{k}\right)$ is given by (3.1) and $U\left(\mathbf{M}_{k}\right)$ by (3.20). Consider the following minimization problem:

$$
\begin{aligned}
\overline{\mathbb{P}}_{k}: & \left(\mathbf{M}_{k}^{0}, \alpha^{0}, \beta^{0}, \delta^{0}\right)= \\
& \arg \min _{\mathbf{M}_{k}, \alpha, \beta, \delta}\left\{\delta \mid\left(\mathbf{M}_{k}, \alpha, \beta, \delta\right) \in \bar{\Omega}\right\}
\end{aligned}
$$

Proposition 5. The minimization problem $\overline{\mathbb{P}}_{k}$ is a linear programming problem.

The problem $\overline{\mathbb{P}}_{k}$ is an LP:

$$
\overline{\mathbb{P}}_{k}: \quad \min _{\gamma}\{\delta \mid \gamma \in \bar{\Gamma}\}
$$

where $\gamma \triangleq\left(\operatorname{vec}\left(\mathbf{M}_{k}\right), \operatorname{vec}\left(\Lambda_{k}\right), \operatorname{vec}\left(\Theta_{k}\right), \alpha, \beta, \delta\right)$ and :

$$
\begin{aligned}
\bar{\Gamma} \triangleq\{\gamma \mid & \sum_{i=0}^{k-1}\left(\eta L_{i} \mathbf{1}_{t}+C_{x} D_{i}\left(\mathbf{M}_{k}\right) f\right) \leq \alpha c_{x}, \\
& -L_{i} \leq C_{x} D_{i}\left(\mathbf{M}_{k}\right) E \leq L_{i}, \quad i \in \mathbb{N}_{k-1}, \\
& \sum_{i=0}^{k-1}\left(\eta T_{i} \mathbf{1}_{t}+C_{u} S_{i} \mathbf{M}_{k} f\right) \leq \beta c_{u}, \\
& -T_{i} \leq C_{u} S_{i} \mathbf{M}_{k} E \leq T_{i}, \quad i \in \mathbb{N}_{k-1}, \\
& (\alpha, \beta) \in[0,1] \times[0,1], \\
& \left.\mathbf{M}_{k} \in \mathbb{M}_{k}, q_{\alpha} \alpha+q_{\beta} \beta \leq \delta\right\}
\end{aligned}
$$

where $\Theta_{k} \triangleq\left\{T_{0}, T_{1}, \ldots T_{k-1}\right\}\left(\right.$ each $\left.T_{i} \in \mathbb{R}^{q_{u} \times t}\right)$ and $S_{i}$ is selection matrix of the form $S_{i}=$ $\left[\begin{array}{lllllll}0 & 0 & \ldots & I & \ldots & \mathbf{0} & \mathbf{0}\end{array}\right]$. It is possible to specify a variety of objective functions by minor modification of the definition of the set $\bar{\Omega}(3.22)$ and still obtain a tractable convex optimization problem. However, an appropriate objective function is the minimization of $q_{\alpha} \alpha, q_{\beta} \beta$ subject to the existence of a RCI set $R_{k}\left(\mathbf{M}_{k}\right)$ for the system (2.1) and constraint set $(\alpha X, \beta U, W)$. The weights $q_{\alpha}$ and $q_{\beta}$ express a preference for relative contraction of the state and control constraint sets. 
The solution $\mathbf{M}_{k}^{0}$ to problem $\overline{\mathbb{P}}_{k}$ (which exists if $\bar{\Omega} \neq \emptyset)$ yields a set $R_{k}^{0} \triangleq R_{k}\left(\mathbf{M}_{k}^{0}\right)$ and feedback control law $\mu^{0}(x)=\mathbf{M}_{k}^{0} \mathbf{w}^{0}(x)$ satisfying $R_{k}^{0} \subseteq$ $\alpha^{0} X$ and $\mu^{0}(x) \in U\left(\mathbf{M}_{k}\right) \subseteq \beta^{0} U$ for all $x \in R_{k}^{0}$. It follows from Theorem 2 and the discussion above that the set $R_{k}^{0}$, if it exists, is RPI for system $x^{+}=A x+B \mu^{0}(x)+w$ and constraint set $\left(X_{\mu^{0}}, W\right)$, where $X_{\mu^{0}} \triangleq \alpha^{0} X \cap\left\{x \mid \mu^{0}(x) \in \beta^{0} U\right\}$. There might exist more than one set $R_{k}\left(\mathbf{M}_{k}\right)$ that yields the optimal cost $\delta^{0}$. The cost function can be modified. For instance, an appropriate choice is a positively weighted quadratic norm of the decision variable $\gamma$ that yields a unique solution, since in this case problem becomes a quadratic programming problem of the form $\min _{\gamma}\left\{|\gamma|_{Q}^{2} \mid \gamma \in\right.$ $\bar{\Gamma}$ \}, where $Q$ is positive definite and it represents the suitable weight. A relevant observation is:

Proposition 6. Suppose that the problem $\overline{\mathbb{P}}_{k}$ is feasible for some $k \in \mathbb{N}$ and the optimal value of $\delta_{k}$ is $\delta_{k}^{0}$, then for every integer $s \geq k$ the problem $\overline{\mathbb{P}}_{s}$ is also feasible and the corresponding optimal value of $\delta_{s}$ satisfies $\delta_{s}^{0} \leq \delta_{k}^{0}$.

If the origin is an interior point of $W$, the condition (3.3) can be replaced by the following condition:

$$
\mathbf{M}_{k} \in \overline{\mathbf{M}}_{k} \triangleq\left\{\mathbf{M}_{k} \mid D_{k}\left(\mathbf{M}_{k}\right) W \subseteq \varphi W\right\}
$$

for $\varphi \in[0,1)$ and $k \geq n$. A family of the sets $R_{(\varphi, k)}\left(\mathbf{M}_{k}\right)$ defined by:

$$
R_{(\varphi, k)}\left(\mathbf{M}_{k}\right) \triangleq(1-\varphi)^{-1} R_{k}\left(\mathbf{M}_{k}\right)
$$

for couples $(\varphi, k)$ such that $(3.26)$ is true, is a family of the polytopic RCI sets:

Theorem 3. Given any couple $\left(\varphi, \mathbf{M}_{k}\right) \in[0,1) \times$ $\overline{\mathbb{M}}_{k}, k \geq n$ and the corresponding set $R_{(\varphi, k)}\left(\mathbf{M}_{k}\right)$, there exists a control law $\mu: R_{(\varphi, k)}\left(\mathbf{M}_{k}\right) \rightarrow \mathbb{R}^{m}$ such that $A x+B \mu(x) \oplus W \subseteq R_{(\varphi, k)}\left(\mathbf{M}_{k}\right), \forall x \in$ $R_{(\varphi, k)}\left(\mathbf{M}_{k}\right)$.

\section{COMPARISON}

A theoretical comparison of the proposed procedure with the previous results is given in (Raković, 2005). The advantages of our method lie in the facts that: (i) hard state and control constraints are incorporated directly into the optimization problem and, (ii) the feedback control law $\mu$ : $R_{k}\left(\mathbf{M}_{k}\right) \rightarrow U$ is piecewise affine function of $x \in$ $R_{k}\left(\mathbf{M}_{k}\right)$. These advantages are illustrated bellow by a numerical example:

$$
x^{+}=\left[\begin{array}{ll}
1 & 1 \\
0 & 1
\end{array}\right] x+\left[\begin{array}{l}
1 \\
1
\end{array}\right] u+w
$$

where $w \in W \triangleq\left\{\left.w \in \mathbb{R}^{2}|| w\right|_{\infty} \leq 1\right\}$. The hard state and control constraints are:

$$
\begin{aligned}
X= & \left\{x \mid-3 \leq x^{1} \leq 1.85,-3 \leq x^{2} \leq 3,\right. \\
& \left.x^{1}+x^{2} \geq-2.2\right\}, U=\{u|| u \mid \leq 2.4\}
\end{aligned}
$$

where $x^{i}$ is the $i^{\text {th }}$ coordinate of a vector $x$. In the first attempt we obtain the closed loop dynamics by applying two linear stabilizing state feedback control laws:

$$
K_{1}=-\left[\begin{array}{ll}
0.72 & 0.98
\end{array}\right], K_{2}=-\left[\begin{array}{ll}
1 & 1
\end{array}\right]
$$

and compute the corresponding sets $F_{\left(\zeta_{K}, s_{K}\right)}^{K}$ by application of Algorithm 1 of (Raković et al., 2005). The computed sets violate the state con-

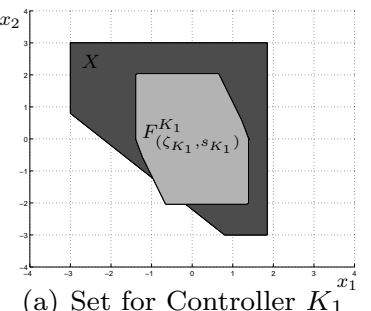

(a) Set for Controller $K_{1}^{x}$

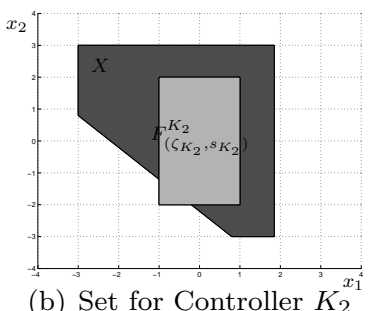

(b) Set for Controller $K_{2}^{x_{1}}$
Fig. 1. Invariant Approximations of $F_{\infty}\left(K_{i}\right)$ : Sets $F_{\left(\zeta_{K_{i}}, s_{K_{i}}\right)}^{K_{i}}, i=1,2$

straints as illustrated in Figure 1. The corresponding control polytopes are:

$U\left(K_{1}\right)=\{u|| u \mid \leq 2.4680\}, U\left(K_{2}\right)=\{u|| u \mid \leq 3\}$,

where $U(K) \triangleq K F_{\left(\zeta_{K}, s_{K}\right)}^{K}$ so that the control constraints are also violated.

By solving the optimization problem $\overline{\mathbb{P}}_{k}$ with the

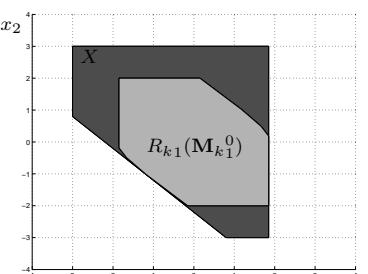

(a) Set for Controller $\mathbf{M}_{k}^{x_{1}^{4}}$

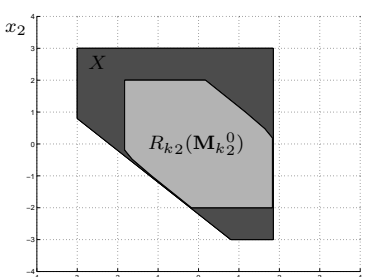

(b) Set for Controller $\mathbf{M}_{k}^{x_{j}^{4}}$
Fig. 2. Invariant Sets $R_{k i}\left(\mathbf{M}_{k i}^{0}\right), i=1,2$

following design parameters:

$\left(k, q_{\alpha}, q_{\beta}\right)_{1}=(5,0,1),\left(k, q_{\alpha}, q_{\beta}\right)_{2}=(5,1,0)$

we computed the following matrices $\mathbf{M}_{k i}^{0}, i=$ 1,2 :

$\mathbf{M}_{k 1}^{0}=\left[\begin{array}{cc}-0.4875 & -1 \\ 0.2199 & 0 \\ 0.1154 & 0 \\ 0.0596 & 0 \\ 0.0926 & 0\end{array}\right], \mathbf{M}_{k 2}^{0}=\left[\begin{array}{cc}-0.5038 & -1 \\ 0.2456 & 0 \\ 0.1132 & 0 \\ 0.0521 & 0 \\ 0.0930 & 0\end{array}\right]$

The sets constructed from the solution of the optimization problem $\overline{\mathbb{P}}_{k}$ satisfy state and control constraints as it can be seen from Figure 2 and from the fact that: 


$$
U\left(\mathbf{M}_{k i}^{0}\right)=\{u|| u \mid \leq 1.975\}, i=1,2,
$$

To make our comparison as fair as possible, we consider also the following two linear state feedback control laws, constructed from the first row of the optimized matrices $\mathbf{M}_{k}$ :

$$
K_{3}=-[0.48751], K_{4}=-\left[\begin{array}{ll}
0.5038 & 1
\end{array}\right]
$$

The corresponding sets $F_{\left(\zeta_{K}, s_{K}\right)}^{K}$ are shown in Figure 3 . The control constraints are satisfied, but the computed sets violate the state constraints.

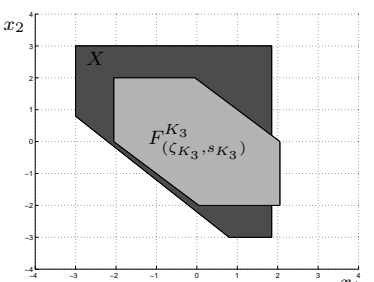

(a) Set for Controller $K_{3}^{x}$

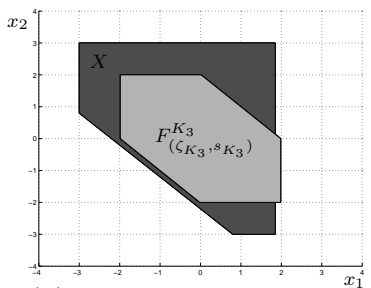

(b) Set for Controller $K_{4}^{x_{1}}$
Fig. 3. Invariant Approximations of $F_{\infty}\left(K_{i}\right)$ : Sets

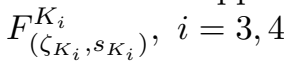

\section{APPLICATIONS AND CONCLUSIONS}

The results of this paper can be used in the design of robust reference governors, predictive controllers and time-optimal controllers for constrained, linear discrete time systems subject to additive, but bounded disturbances.

The main contribution of this note is a novel characterization of a family of polytopic robust control invariant sets for which the corresponding control law is non-linear (piecewise affine) enabling better results to be obtained compared with existing methods where the control law is linear. Construction of a member of this family contained in the minimal $p$-norm ball or reference polytopic set can be obtained from the solution of an appropriately specified LP. The optimized robust control invariance algorithms were illustrated by an example, in which significant improvements over existing methods was illustrated.

The results can be extended to the case when disturbance belongs to an arbitrary polytope. Moreover, it is also possible to extend the results to the case when the system dynamics are parametrically uncertain.

\section{ACKNOWLEDGMENT}

The authors gratefully acknowledge the useful feedback and suggestions provided by Professors R. B. Vinter and E. De Santis, and Dr P. Grieder. All set computations were performed with the Geometric Bounding Toolbox (Veres, 2003).

\section{REFERENCES}

Bemporad, A. and M. Morari (1999). Robustness in Identification and Control. Chap. Robust model predictive control: A survey, pp. 207226. Number 245 In: Lecture Notes in Control and Information Sciences. Springer-Verlag. Survey paper.

Bertsekas, D. P. and I. B. Rhodes (1971). On the minimax reachability of target sets and target tubes. Automatica 7, 233-247.

Blanchini, F. (1992). Minimum-time control for uncertain discrete-time linear systems. In: Proc. 31st IEEE Conference on Decision and Control. Vol. 3. Tuczon AZ, USA. pp. 262934.

Blanchini, F. (1999). Set invariance in control. Automatica 35, 1747-1767. survey paper.

Findeisen, R., L. Imsland, F. Allgöwer and B. A. Foss (2003). State and output feedback nonlinear model predictive control: An overview. European Journal of Control 9(2-3), 190-206. Survey paper.

Gilbert, E. G. and I. Kolmanovsky (1999). Fast reference governors for systems with state and control constraints and disturbance inputs. International Journal of Robust and Nonlinear Control 9(15), 1117-41.

Horn, R. A. and C. R. Johnson (1985). Matrix Analysis. Cambridge University Press, UK.

Kolmanovsky, I. and E. G. Gilbert (1998). Theory and computation of disturbance invariance sets for discrete-time linear systems. Mathematical Problems in Engineering: Theory, Methods and Applications 4, 317-367.

Langson, W., I. Chryssochoos, S. V. Raković and D. Q. Mayne (2004). Robust model predictive control using tubes. Automatica 40, 125-133.

Mayne, D. Q. (2001). Control of constrained dynamic systems. European Journal of Control 7, 87-99. Survey paper.

Mayne, D. Q. and W. R. Schroeder (1997). Robust time-optimal control of constrained linear systems. Automatica 33, 2103-2118.

Raković, S. V., E.C. Kerrigan, K.I. Kouramas and D. Q. Mayne (2005). Invariant approximations of the minimal robust positively invariant set. IEEE Transactions on Automatic Control 50(3), n.a.

Raković, Saša V. (2005). Robust Control of Constrained Discrete Time Systems: Characterization and Implementation. PhD thesis. Imperial College London, London, United Kingdom.

Veres, S. M. (2003). Geometric Bounding Toolbox (GBT) for Matlab. Official website: http://www.sysbrain.com. 